\title{
APUNTES SOBRE PROCESOS FORMATIVOS Y PRÁCTICAS CREATIVAS EN DANZA
}

\section{Sketches on formative processes and creative practice in dance}

\author{
Virgínia Cortés Ramos ${ }^{1}$ (Vicky Cortés Ramos) \\ Universidad Nacional - UNA | Costa Rica
}

Resumen: El presente trabajo busca dialogar en torno a la manera en que se ha transformado la interacción entre formación y creación artística contemporánea en los procesos formales de educación, a partir de un breve análisis de las lógicas y tensiones paradigmáticas que se manifiestan en las universidades y la necesidad de impulsar dinámicas formativas abiertas, plurales, enactivas.

Palabras Claves: Danza, Creación, Formación.

Resumo: O presente trabalho busca discutir sobre a maneira como a interação entre formação e criação artística contemporânea vem se transformando no contexto dos processos formais de educação. Para tanto, parte-se de uma breve análise das lógicas e tensões paradigmáticas que se manifestam nas universidades e da necessidade de promover dinâmicas de treinamento abertas, plurais e enativas.

Palavras-chave: Dança; Criação; Formação.

Abstract: This article wishes to propose a dialogue concerning the way in which the interaction between formative processes and contemporary artistic creation is transformed in the context of formal education, based on the analysis of paradigmatic propositions and tensions within the universities, and the necessity for the development of open, plural, and enactive formative dynamics.

Keywords: Dance; Creation; Education.

\footnotetext{
${ }^{1}$ Maestría Profesional en Formación Dancística y Coreografía. Escuela de Danza, Universidad Nacional de Costa Rica (UNA).
}

Revista Moringa - Artes do Espetáculo, João Pessoa, UFPB, v. 9 n. 1, jan/jun 2018, p. 23 a 30

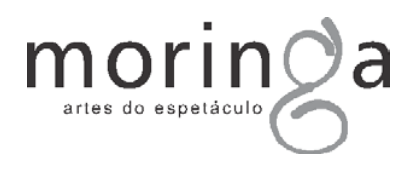


"que veía a través de sus ojos no con ellos"

(William Blake)

Sabemos el valor de lo que significa la incorporación de la enseñanza de la danza, no sólo en las escuelas superiores de arte o las universidades, sino en cualquier ámbito de la educación formal. Como lo señalaba férreamente Laban en la introducción de su libro Danza Educativa Moderna: “¿Cómo procederemos? ¿Ofrecen los vestigios del histórico arte de la danza una base adecuada sobre la cual construir la nueva enseñanza de la danza en la escuela, o necesitamos, en nuestra compleja civilización moderna, de un nuevo enfoque del problema?" (LABAN, 1975, p. 13). Considero que deberíamos volver a formular la pregunta que él se hacía, pero desde el lugar en que nos encontramos ahora.

En este artículo quisiera referirme al contexto de las escuelas de arte, en particular desde la danza (sobre todo las que han conservado la escolástica tradicional), articulada a la noción de formación-creación que, ineludiblemente, atraviesa también el inmenso tema de los modelos educativos.

De entrada, hay que considerar que el hecho escénico, como expresión artística, se basa intrínsecamente en una articulación entre sus diversos componentes expresivos. La separación, analítica o práctica, de este hecho en componentes disciplinarios especializados con intención formativa- sólo tiene sentido en ciertos aspectos del proceso creativo (por el tipo de formación especializada que generalmente tienen las personas involucradas), pero en el resultado final cada componente está fusionado con los demás, y los límites entre los aportes disciplinarios son prácticamente indisolubles.
Además, es relativamente reciente el fenómeno de la especialización disciplinaria, sobre todo en Occidente, en donde se produce un nivel de autonomización de la práctica dancística. Así, aunque la creación es la fuente primera para forjar la ideación dancística y todas sus posibles ramificaciones (incluyendo la técnica), en el ámbito formativo se excluye la interacción con la experiencia de la persona como individuo, y más aún la colaboración entre personas.

Desde las culturas tradicionales hasta nuestros días este proceso de autonomización disciplinar, que impone la metodología de "lo formativo" aislada de la experiencia enactiva, término planteado por Francisco Varela (1990), ha tendido a crear una cierta desintegración, una dicotomía entre el valor que se le da a lo que se asimila desde la información externa y el valor que se le da a lo que se percibe desde la propia experiencia. Cesar Ojeda explica en relación con el término "enacción" utilizado por Varela:

Lo que en suma desea enfatizar es la creciente convicción de que la cognición no es la representación de un mundo predado por una mente pre-dada sino más bien la puesta en obra de un mundo y una mente a partir de una historia de la variedad de acciones que un ser realiza en el mundo. (OJEDA, 2001, párrafo 16)

¿Cómo no tomar en cuenta la ineludible y voluble condición del lo particular como clave, aceptando el espacio formativo como un proceso plural y multifacético $\mathrm{y}$, sobre todo, contextualizado en nuestra contemporaneidad?

El arte contemporáneo, debemos agregar, está en un movimiento vigoroso de constantes recontextualizaciones, como nos señala García Canclini: "La historia contemporánea 
del arte es una combinación paradójica de conductas dedicadas a afianzar la independencia de un campo propio y otras empecinadas en abatir los límites que lo separan." (CANCLINI, 2010, p. 15).

Aun comprendiendo la diversidad del panorama, esto no significa que dejemos de reconocer que la autonomización disciplinar y las informaciones organizadas que contemplan lo formativo, pueden posibilitar nuevas búsquedas y lógicas de trabajo práctico, según las diversas necesidades creadoras.

\section{De cómo pensamos como pensamos}

Las interrogantes que se han planteado nos llevan a un escenario complejo con diversas visiones en tensión: por un lado, está el paradigma que surge desde la modernidad y que se desarrolló sobre la base del pensamiento cartesiano; por otro, están los nuevos paradigmas que enfocan el abordaje desde la complejidad y la interacción.

Esta tirantez se siente con fuerza en el marco de la formación, la investigación y la creación artística en las universidades, y por eso es importante señalar algunas características básicas del "patrimonio" paradigmático dominante.

El modelo cartesiano plantea la necesidad de una imparcialidad. Subliminalmente su impulso está en la negación de la incertidumbre, y es desde esta particular forma de buscar "la verdad" que se ha construido toda una lógica mecanicista, unívoca, lineal. Uno de sus resultados es la especialización y la fragmentación como enfoque analítico, que ha permeado la educación sin excepción. En este sentido Denise Najmanovich apunta: "El mundo llamado "objetivo" es un mundo muy alejado de la experiencia humana, es un mundo inventado por un sujeto que se piensa a sí mismo como "observador neutro" (NAJMANOVICH, 2005, p. 31).

Esto no es poca cosa, porque independientemente de sus grandes logros 0 desaciertos, este "invento" ha tenido el poder de imponer acuerdos sobreentendidos que nos hacen buscar y pensar de una determinada manera.

No obstante el dominio de la visión cartesiana, han surgido, dentro de la misma tradición científica, respetables voces señalando la necesidad de una absoluta renovación paradigmática. En este sentido David Bohm apunta:
Al concentrarnos en este tipo de análisis y dividir constantemente los problemas en distintas áreas especializadas, vamos ignorando el contexto más amplio que da a las cosas su unicidad. De hecho, ese espíritu se está extendiendo ahora más allá de la ciencia, no sólo a la tecnología sino a nuestra visión general de la vida como un todo. Conocer se valora en la actualidad como la posesión de los medios para predecir, controlar y manipular las cosas. (BOHM, 1998, p. 53)

La separación y división, como metodología de estudio e investigación para "comprender las cosas", produce certezas recalcitrantes que, "huyendo" de la fe, nos termina devolviendo a ella, ya que funciona como una camisa de fuerza que no permite la aceptación de otras formas de producir conocimiento, de entender y de transformarnos. En la universidad tradicional, toda la lógica académica, burocrática y administrativa está sostenida desde este orden, que subsiste no sin cuestionamientos. 
Najmanovich se refiere a lo cartesiano y su impacto en la percepción de lo corporal en su momento histórico:

[La] revolución cartesiana ha tenido $-y$ tiene todavía - implicancias fundamentales para el pensamiento de la corporalidad (...) [D]a alta prioridad a lo "visible" -en tanto fuente de representación sistemática-, a lo reproducible - en tanto aporta una forma de eliminar incertidumbres-, a lo mecánico y previsible en tanto permite la manipulación-. (NAJMANOVICH, 2005, p. 33)

Pero a pesar de la fuerza de la costumbre en cuanto a la necesidad de certidumbre, poco a poco se comienzan a movilizar las cadenas profundamente anquilosadas del paradigma tradicional. En este sentido, Bohm reflexiona sobre la naturaleza de la creatividad en la ciencia:

(...) para comprender la naturaleza esencial del cambio en la ciencia, [es] necesario ver cómo surgen de hecho nuevas ideas a pesar de esta rigidez. Al hacerlo así podremos introducirnos en la naturaleza de la creatividad, sin la cual la ciencia se vería apresada de manera indefinida en las "vías inflexibles" que ella misma ha trazado con su propio progreso en el pasado. (BOHM, 1998, p. 71)

La "fe ciega" en una metodología desde una sola ruta del "pensar", donde suponemos se registran certezas comprobables, limita la comprensión compleja de la existencia. ¿Cómo podemos avanzar si nos sostenemos solamente desde una experiencia cronificada en el tiempo que repetimos sistemáticamente? Najmanovich afirma: "Se trata entonces de construir una gramática centrada en la acción, en la producción, y no en el producto o en el objeto" (NAJMANOVICH, 2005, p. 14).
Y para enfrentar los retos de transformación debemos estar muy conscientes de cómo se expresa lo cartesiano en la formación e investigación del cuerpo en la danza, ya que las imposiciones modélicas y hegemónicas de larga data, interactúan, de una u otra manera, en las formas en que se institucionalizan las prácticas artísticas que se construyen según la concepción del conocimiento que se trae. $Y$ esto afecta de diferentes maneras el desarrollo de visiones y, por supuesto, las experiencias particulares. En concreto, podríamos decir que hay un énfasis en las técnicas sistematizadas, no como formas de interactuar, sino como procedimientos y modelos a imitar. $Y$ se ha producido una estandarización del conocimiento técnico y del oficio a espaldas de las particularidades de las necesidades creadoras.

Cuando vemos la técnica o la destreza como "algo" que hay que lograr, caemos otra vez en la dicotomía entre "práctica" y "perfecto", lo cual nos conduce a cualquier cantidad de círculos viciosos. Si improvisamos con un instrumento, herramienta o idea que conocemos bien, tenemos la sólida técnica para expresarnos. Pero la técnica puede tornarse demasiado sólida... es posible que nos acostumbremos tanto a saber como hay que hacerlo que nos alejamos de la novedad de la situación de hoy. Este es el peligro inherente a la competencia misma que adquirimos con la práctica. La competencia que pierde el sentido de sus raíces en el espíritu juguetón se oculta tras una rígida forma de profesionalismo. (NACHMANOVITCH, 2009, p. 85)

\section{Trazando nuevos caminos: creación/aprendizaje y cuerpo}

El acto creador, como experiencia pedagógica fundacional, permite que el estudiante genere su búsqueda y sentido. Se trata, en resumen, 
de cambiar de un modo fijado en objetivos de enseñanza preestablecidos, competencias, etc., a otro que toma como su principal condición el aprendizaje. Por eso es vital, entre docentes y estudiantes, una transformación de la noción de la enseñanza institucionalizada, administrativista, en aras de una comunicación al interior de una relación participativa.

Los principios de la interacción creadora en los procesos de aprendizaje no sólo tienen un sentido de carácter metodológico/vivencial, mas también involucra la posibilidad de la construcción del oficio artístico que trascienda un campo disciplinario único, incorporando la experiencia y los impulsos desde prácticas colectivas y plurales.

La danza, como expresión, surge desde el estado vital del ser. El "cuerpo vivo" no es un "instrumento" deshabitado: está atravesado por lo que vivimos $y$, por ello, se encuentra necesitado -al menos desde la danza contemporánea- de buscar esa "forma coherente" de su momento vital. Esta realidad, que nace del acto creador, es marginada por la academia, como nos señala Najmanovich:

La objetividad supone la capacidad de unos sujetos para abstraerse. Es decir, para suponer que ni su corporalidad -que incluye tanto su peculiaridad perceptiva como emocional y su forma de acción en el mundo- ni su subjetividad, ni los vínculos que establece, influyen en el conocimiento del mundo. Desde esta perspectiva conceptual, el cuerpo es aquello que puede ser medido, así como el cuerpo de la perspectiva era aquel que puede ser representado. (NAJMANOVICH, 2005, p. 32)

Y respecto al "dominio controlador de percepción corporal", que también influye en las estéticas del cuerpo, apuntala Najmanovich:
(...) el cuerpo es un mecanismo: sustancia externa regida por leyes, donde cada efecto es un productor necesario de una causa. Aquello que en la filosofía ha dado en llamarse "el problema cuerpo-mente" no existía antes de este giro cartesiano y es un producto exclusivo que nace al calor de las premisas dualistas. Éstas, a su vez, se originan en los procesos de estandarización sociales y tecnológicos, que permiten la generación de procedimientos estables, normativizados, repetibles y predecibles, que parecen ser independientes de los sujetos que los llevan a cabo. (NAJMANOVICH, 2005, p. 33)

El cuerpo no "se integra" desde la teoría, sino según lo que necesita y puede incorporar en su vivir. Esta noción comienza a dar sus frutos en múltiples formas de atender la visión de nuestro cuerpo alejada de una relación mecanicista, reproductora y representacional. Desde los nuevos paradigmas de la ciencia, del arte y de la educación, que integra la cognición, la creación, la improvisación y la somática e infinidad de transversalidades, es posible avanzar en un conocimiento que moviliza el anquilosamiento perceptivo del cuerpo.

En este sentido, debemos preguntarnos: ¿Cuál podría ser la dinámica de la universidad con relación al mundo de la danza, atravesado por grandes cambios estructurales y múltiples vías en constante ebullición? Y, sobre todo, ¿cuál es nuestro aporte a la educación desde nuestra forma de pensar/hacer en las escuelas de arte y, en particular, las escuelas de danza?

\section{Enacción e improvisación}

Aquí me parece importante recuperar la noción de enacción e introducir el tema de la improvisación como posibilidad de movimiento que hace emerger al sujeto. En este sentido Ann Cooper Albright y David 
Gere, en su libro Taken by surprise, afirman:

De hecho, si el dualismo cartesiano del cuerpo como algo separado de la mente se va a superar alguna vez -como parece ser la tendencia principal de los estudios dancísticos actuales- la improvisación proporciona el paradigma perfecto. Porque es mientras se improvisa que la inteligencia del cuerpo se manifiesta de manera más ineluctable, y que una mente ágil se convierte en una necesidad. ${ }^{2}$ (COOPER, GERE, 2003, p. XIV)

No podemos tratar al cuerpo como mecanismo de ajuste. Lo somático, por ejemplo, incorpora la experiencia personal: no la relega, trabaja con ella. Poner atención a lo que hacemos no pasa por dejar "estacionada", fuera del estudio, la vida interior. La improvisación e incluso la técnica más rigurosa no podría "encarnarse" sin esa integridad consciente y corporal.

La improvisación (entendida como forma de conocimiento) en una escuela de arte con un sentido epistémico convencional, puede parecer un tema "poco académico", sobre todo porque no se puede abarcar la condición unívoca del fenómeno para teorizarlo. La experiencia creadora, 0 la experiencia improvisatoria, nos empuja a márgenes de argumentos intangibles (que, por otro lado, no debería confundirse con un sentido enigmático, sino más bien, ligada a una articulación cuidadosa del hacer en la experiencia).

Con todos los condicionamientos epistémicos, cuesta imaginar que la improvisación para la experiencia creadora sea, en realidad, una condición cognitiva ${ }^{3}$ muy particular y rigurosa

\footnotetext{
2 Traducción libre: Vicky Cortés.

${ }^{3}$ En el sentido que Varela expone: "El ser cognitivo es la manera en que el organismo, mediante su actividad
}

de esfuerzo en el ser humano. No obstante, la cognición y la enacción exigen la rapidez, vivacidad y destreza de una resolución inmediata como requisito de juego. (Los niños se desarrollan con facilidad a través de esta sofisticada capacidad de enactuar.)

El hecho de no tener "consciencia", o una programación cierta de lo que deviene, no significa que crear e improvisar "sea cualquier cosa" a partir de un paisaje "desierto", sin configuración. Siempre se está suscrito a un mundo de experiencias vividas, desde una cultura, una educación, así como al complejo diseño de nuestra naturaleza organizada y de nuestra condición enactiva. Desde un lugar muy esencial, en cuanto a la experiencia de "ser", Varela, reflexiona:
Se trata de la emergencia de un nivel global que surge a partir de las reglas locales y que tienen un estatus ontológico diferente, porque trae consigo la creación de un individuo, o de una unidad cognitiva. Entonces, cuando ven a un animal en movimiento, o me ven a mí dirigiéndoles la palabra, me comporto como una unidad coherente, no como una mera yuxtaposición de movimiento, voz, mirada, y postura. Soy una unidad integrada, más o menos armónica a la que denomino "mi mismo" (VARELA, 2002, p. 244)

La rigidez encauzada en la forma en cómo nos educamos, y en cómo pensamos y percibimos, fija ese aprendizaje en la memoria, obstaculizando la experiencia. Nos concebimos como una serie limitada de problemas a resolver en la "mente".

\footnotetext{
autoproducida, llega a ser una entidad distinta en el espacio, aunque siempre acoplada a su correspondiente medioambiente, del cual, no obstante, será siempre diferente. Un ser distinto coherente que, por el proceso mismo de constituirse (a si mismo), configura un mundo externo de percepción y acción" (VARELA, 2002, p. 66)
} 
El razonamiento, como forma de conocimiento, proviene de argumentos, explicaciones, deducciones, juicios, conceptos; en cambio el conocimiento intuitivo abarca todo nuestro conocimiento y la experiencia de lo que somos.

Se trata de ponernos en un estado de apertura desprejuiciada, entrando al juego donde "nunca se pierde", que nos permita descubrir, no encubrir, lo que consideramos una "limitación". Podemos embarcar en una exploración de encuentros sin costumbre, insólitos, para movilizar nuestro engramas, y ligarlo también a nuestra reflexión en el desarrollo técnico que se confronta con nuevos "problemas corporales" para resolver, y de nuestro pensamiento en la creación estética. Se trata de asumir un estado de atención en nuestro autoconocimiento. Así, las "metodologías" de improvisación y creación se pueden reestructurar, re-componer, re-configurar, resignificar, re-contextualizar, pero su principio es ese lugar sin tiempo.

\section{A manera de conclusión}

Hablar del cuerpo es hablar de lo inasible, porque su existencia es experiencia única e irrepetible. Por esto es importante aproximarse al lugar de su ubicación paradigmática en el contexto actual, y comprender que son múltiples los factores que intervienen y generan la experiencia desde el cuerpo. Hay que abordar la cognición (entendida a partir del concepto desarrollado por Varela) desde la complejidad que amerita, y no circunscribirlo, de manera segmentada, como "objeto de estudio", pensando en una imparcialidad "aparente" que lo resguarda en la noción de certidumbre construida en la visión de nuestra cultura. El privilegiar una forma de comprender el mundo trajo esta noción de la mente separada del cuerpo, del conocimiento separado de la experiencia; como si el conocer fuera un fenómeno externo.

El educador somático de Yvan Joly nos dice:

(...) cualquier forma de acción en el mundo, cualquier operación, sea concreta o abstracta, sucede con el cuerpo, en el cuerpo o, aun mejor, como un cuerpo. La inteligencia somática se refiere no solo al uso de nuestro cuerpo para actuar en el mundo, sino también a la base corporal de todos nuestros pensamientos, sensaciones, y sentimientos; se refiere, a final de cuentas, a nuestra propia existencia de estar vivos como personas, únicas e irrepetibles; por ello, es aquella riqueza aún no descubierta de nuestra persona. (JOLY, 2010, p. 3)

Por tanto, no podemos definirnos desde una lógica lineal. La experiencia del cuerpo es multidimensional. El soma es el cuerpo vivido desde el interior, no animado por la mente.

De nuevo Joly nos dice: "La mente está incorporada; el cuerpo tiene mente... una comprensión y un enfoque práctico de la persona como un sistema viviente incorporado $y$ consciente debería disipar el guion del cuerpomente y idejar que la ecuación se disuelva!" (JOLY, 2010, p. 13).

En el análisis antropológico se reconoce que el cuerpo y el movimiento están construidos desde condiciones culturales y patrones de organización de larga data.

Hay que subrayar, además, que en este continuo de aprendizajes quedan por fuera infinidad de historias del cuerpo que no fueron tomadas en cuenta. Se ha negado el reconocimiento y la aceptación de la multiplicidad 
de cuerpos expresivos, y se ha sostenido una visión de mundo eurocéntrica que, aun en este momento, se vive como un roce de fuerzas y un juego de adaptaciones-inadaptaciones con el statu quo, para "permitimos" pertenecer -desde algún escenario- al "patrimonio legitimado" del arte.

Necesitamos, entonces, visibilizar el movimiento y la expresión dinámica de aquella "otra historia": la nuestra, aquí y ahora.

Considerando lo anterior y volviendo a un terreno más concreto, es necesario proponer, por un lado, distribuciones y relaciones más equitativas de la información preestablecida y, por otro, facilitar el recorrido de la experiencia enactiva del aprendizaje, transformando la óptica vigente desde donde establecemos la noción de cognición: mente y cuerpo separados (y donde, además, la mente está en la cabeza). Al respecto, Varela nos dice:

Uno de los más importantes avances en ciencia en los últimos años es la convicción de que no podemos tener nada que se asemeje a una mente o a una capacidad mental sin que esté totalmente encarnada 0 inscrita corporalmente, envuelta en el mundo. Surge como una evidencia inmediata, inextricablemente ligada a un cuerpo que es activo, que se mueve y que interactúa con el mundo. (VARELA, 2002, p. 240)

Si el arte ha sido, precisamente, una forma diferente de "pensar" y "expresar" la experiencia con el mundo, ¿por qué condicionarlo? En otras palabras, ¿por qué sujetar la posibilidad de la experiencia de lo que el educador somático Yvan Joly (2010) llama el "cuerpo viviente"? Estas preguntas, para los procesos de enseñanza-aprendizaje en el arte $-y$ ni qué decir de un contexto creador en donde "el cuerpo se expresa en un lenguaje"- son, desde luego, fundamentales. Pormenorizar respecto a la experiencia como conocimiento nos permite revalorizar su denigrada condición en la episteme tradicional, y potenciarla como fuente de aprendizaje fundamental.

Recebido em:08/05/2018

Aceito em:20/052018

\section{Referências Bibliográficas}

BOHM, D. La totalidad y el orden implicado. Buenos Aires: Editorial Kairós, 1998.

CANCLINI, N. La sociedad sin relato. Antropología y estética de la inminencia. Buenos Aires: Katz Editores, 2010.

COOPER, A. y GERE, D. Taken by surprise. Advanced improvisation reader. Connecticut: Wesleyan University Press, 2003.

JOLY, Y. Educación Somática. Reflexiones sobre la práctica de la conciencia del cuerpo en movimiento. Tlanepantla de Baz. Facultad de Estudios Superiores Iztacala, 2010.

LABAN, R. Danza educativa moderna. Barcelona: Ediciones Paidós, 1975.

NACHMANOVITCH, S. Free Play. La improvisación en la vida y en el arte. Barcelona, Buenos Aires, Mexico: Paidós, 2009.

NAJMANOVICH, D. El juego de los vínculos. Subjetividad y redes: figuras en mutación. Buenos Aires: Editorial Biblos, 2005.

OJEDA, C. Francisco Varela y las ciencias cognitivas. Revista chilena de neuropsiquiatría, Santiago de Chile, v. 39, n. 4, p. 286-295, oct. 2001. ISSN: 0717-9227.

VARELA, F. El fenómeno de la vida. Santiago de Chile: Dolmen Ediciones, 2002. 